\title{
Quantum Information Processing with Superconducting Nanowire Single-Photon Detectors
}

\author{
Takashi YAMAMOTO $^{\dagger, \dagger \dagger a)}$, Nonmember
}

SUMMARY Superconducting nanowire single-photon detector (SNSPD) has been one of the important ingredients for photonic quantum information processing (QIP). In order to see the potential of SNSPDs, I briefly review recent progresses of the photonic QIP with SNSPDs implemented for various purposes and present a possible direction for the development of SNSPDs.

key words: superconducting nanowire single-photon detector, quantum information processing, quantum optics

\section{Superconducting Nanowire Single-Photon Detector}

In the last two decades, detecting the light at the singlephoton level with superconducting devices has been rapidly developed, and its applications have widely spread to many fields: not only quantum information but also sensing and fundamental physics [1]. Although the specification requested to the photon detectors depends on the applications, the typical interesting parameters are (1) response wavelength range, (2) quantum efficiency, (3) dark count rate, (4) timing resolution or jitter, (5) maximum count rate, (6) after pulse rate, and (7) photon number resolution. Furthermore, the performance of the overall detection system including optical fiber input and the cryostat is important. At this moment, in most of the parameters, the photon detectors based on superconducting devises surpass the semiconductor avalanche photodiodes if the use of cryostat is allowed for the applications.

Two types of superconducting detectors, which are named as superconducting nanowire single-photon detector (SNSPD) and superconducting transition edge sensor (TES), have been developed so far. Both two detectors have very high performances in most of the parameters, for example the quantum efficiency approaches to unity and the dark count rate can be the order of Hz. Significant differences appear in (4) the timing resolution and (7) photon number resolution. Typically, SNSPD has a high timing resolution of the order of $\sim$ ps, whereas $\sim$ ns for TES. On the other hand, TES can discriminate the photon number within a temporal mode, but the single SNSPD tell us the arrival

Manuscript received September 3, 2018.

Manuscript revised October 25, 2018.

$\dagger$ The author is with Faculty of Graduate School of Engineering Science, Osaka University, Toyonaka-shi, 560-8531 Japan.

${ }^{\dagger \dagger}$ The author is also with Faculty of Quantum Information and Quantum Biology Division, Institute for Open and Transdisciplinary Research Initiatives, Osaka University, Toyonaka-shi, 5608531 Japan.

a) E-mail: yamamoto@mp.es.osaka-u.ac.jp

DOI: $10.1587 /$ transele.2018SDI0002 of photons, similar to the semiconductor avalanche photodiodes (APD). A recently demonstrated multi-pixel SNSPD array analogous to the CCD camera will have the ability for counting and/or discriminating the number of the arrival photons [2]. In this paper, I briefly review the SNSPD based photonic quantum information studies. Especially, I focus on the usefulness of the high timing resolution, the low dark count rate and the high quantum efficiency in telecom wavelength range of the SNSPDs.

\section{Photonic Quantum Information Processing}

In the optical frequency range of several hundred $\mathrm{THz}$, the thermal radiation at the room temperature is almost negligible even for the experiment performed at the single photon level. Thus the photonic quantum systems are one of the most promising media for quantum information processing. Especially for quantum communication, no medium is competitive with the photonic systems as a carrier of quantum information.

Quantum key distribution (QKD) [3], [4], which allows to generate/amplify the secret key between two legitimate users through the distributed photonic quantum states, is one of the major applications of quantum communication. Vast number of QKD experiments have been done so far, and the achievable lengths have reached $\sim 400 \mathrm{~km}$ [5], [6]. The limitation of the achievable distance is caused by the error rate coming from the disturbance of the transmitted quantum state and the dark counts of the single-photon detectors. Especially, SNSPDs contribute to the reduction of the dark count rate of the single-photon detectors.

The extremely low thermal noise property in the optical frequency range is advantageous not only for the long distance quantum communication, but also for the quantum information processing at the room temperature. The most elemental protocol is the quantum teleportation including Bell measurement [7]. Quantum teleportation is a way to transmit a quantum state from one party named as Alice to the other party named as Bob on the condition that they share entangled quantum systems in advance as shown in Fig. 1 a. Suppose Alice (Bob) has qubit A (B), and the composite system $\mathrm{A}$ and $\mathrm{B}$ is in state $\left|\Psi^{(-)}\right\rangle_{A B}=$ $\left(|0\rangle_{A}|1\rangle_{B}-|1\rangle_{A}|0\rangle_{B}\right) / \sqrt{2}$, which is named as a Bell state. Alice has another qubit $A^{\prime}$ in a state $|\phi\rangle_{A^{\prime}}$ intended to be sent to Bob. Alice performs the projection measurement on two qubits $\mathrm{A}$ and $\mathrm{A}^{\prime}$ with Bell basis, i.e., a set consists of $\left|\Psi^{(-)}\right\rangle_{A A^{\prime}},\left|\Psi^{(+)}\right\rangle_{A A^{\prime}}=\left(|0\rangle_{A}|1\rangle_{A^{\prime}}+|1\rangle_{A}|0\rangle_{A^{\prime}}\right) / \sqrt{2}$, and 
a

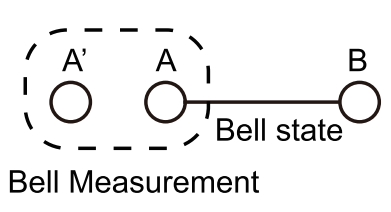

b

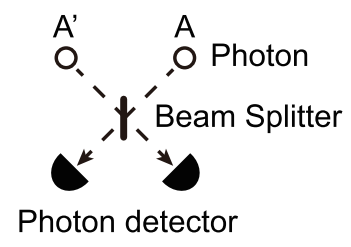

C

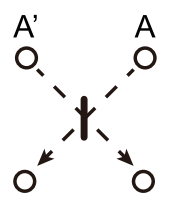

d

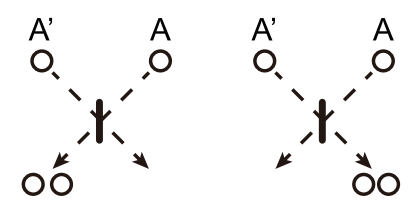

Fig. 1 Schematics of a quantum teleportation and $\mathbf{b}$ photonic Bell measurement. $\mathbf{c}$ The output photon distribution in the case of state $\left|\Psi^{(-)}\right\rangle_{A A^{\prime}}$. d The output photon distribution in the case of the other Bell states $\left|\Psi^{(+)}\right\rangle_{A A^{\prime}}$ and $\left|\Phi^{( \pm)}\right\rangle_{A A^{\prime}}$.

$\left|\Phi^{( \pm)}\right\rangle_{A A^{\prime}}=\left(|0\rangle_{A}|0\rangle_{A^{\prime}} \pm|1\rangle_{A}|1\rangle_{A^{\prime}}\right) / \sqrt{2}$. The projection measurement on two qubits is named as Bell measurement. The results of the Bell measurement is represented by 2 bits, which are sent to Bob. Depending on the received 2-bit information, Bob performs one of four unitary transformations on his qubit $\mathrm{B}$, resulting in the state $|\phi\rangle$ on qubit B. In the quantum teleportation protocol, with the use of the shared entangled state as a resource, an arbitrary qubit state is transmitted from Alice to Bob by the 2-bit classical communication. The 2-bit information announced to Bob is independent on the state of qubit A' and the shared Bell state has no correlation with any third parties. Thus the state transmission by the quantum teleportation can be used to perform QKD.

In the photonic quantum information processing, the key element of the quantum teleportation: Bell measurement on two qubits was demonstrated for the first time by a beam splitter and photon detectors [8]. In the experiment, entangled photons $\mathrm{A}$ and $\mathrm{B}$ in the polarization degree of freedom have been generated by the spontaneous parametric down conversion (SPDC) [9]. The additional photon A' in a polarization state was prepared by the projection measurement on a photon in a polarization basis of an entangled photon pair. Photon A and A' are mixed at a beam splitter and measured by the photon detectors placed at each output port as shown in Fig. 1 b. When the state of photon $\mathrm{A}$ and $A^{\prime}$ is one of the Bell state $\left|\Psi^{(-)}\right\rangle_{A A^{\prime}}$, then the photon appears in each output port simultaneously in Fig. $1 \mathrm{c}$. In the residual cases where the other Bell states in A and A', two photons appear in one of the output ports due to the well-known Hong-Ou-Mandel (HOM) interference [10] in Fig. $1 \mathrm{~d}$. If the polarization of two photons in the same output port is orthogonal each other, the state of photon $\mathrm{A}$ and A' is another Bell state $\left|\Psi^{(+)}\right\rangle_{A A^{\prime}}$. Thus, even with a beam splitter, Alice can project the state onto a half of Bell states at least. It is proven that the quantum teleportation with such linear optics cannot succeed with unit probability. However the significant property of the quantum teleportation is not only the deterministic success but also the conclusive success: a non-demolition state selection with the notice of the success. This property has an important role in photonic QIP, because even if the arrival of the photon A' is stochastic, the success of the teleportation of the survived photon can be noticed by the success of the Bell measurement. In order to implement high fidelity and efficiency experiments, the performance of the photon detectors used for Bell measurement is important.

In the process of the quantum teleportation, the Bell state used as a resource replaces the direct transmutation of the quantum state by the 2-bit classical communication. Along with this idea, different resources and operations are expected to provide other useful quantum information tasks [11]-[17]. A general formalism for such tasks is the one-way quantum computation [18] or the measurementbased quantum computation with a large cluster state [19]. In this formalism, thanks to the entanglement of the cluster state, any quantum operation is performed with a singlequbit operation and measurement, which is suitable for photonic QIP. In principle, the large scale entanglement resources can be generated by a small scale resources such as multiple single-photon states with linear optics and photon detectors. Therefore, to scale up the size of resources, integrated optical circuits based on silicon photonics, optical waveguide circuits and frequency multiplexing have been actively studied [20]-[26]. The direct generation of the resource states has also been developed recently [27]-[29]. It is also important to develop a large scale single-photon detector array with SNSPD suitable for such optical circuits and multiplexing [30].

\section{Timing Measurement on Photonic QIP}

Many photonic quantum information processings including quantum teleportation, entanglement swapping, and measurement-device-independent (MDI) QKD employ quantum interference among multiple photons. In the case of photonic quantum teleportation experiment, Bell measurement utilizes HOM interference of two photons. In the standard HOM interference experiment, photon sources are synchronized with the pulsed excitation of SPDC, which ensures that independent photons simultaneously arrive at the beam splitter used for HOM interference. The schematic of the experiment is shown in Fig. 2. In order to obtain a high visibility of HOM interference, the coherence time $\left(T_{\mathrm{c}}\right)$ of each photon should be much larger than the pulse width $\left(T_{\mathrm{p}}\right)$ of the pump light, which determines ambiguity of the arrival time of the photons at the beam splitter. When the required condition $T_{\mathrm{c}} \gg T_{\mathrm{p}}$ is satisfied, the timing resolution $\left(T_{\mathrm{d}}\right)$ of the photon detectors used for the observation of HOM interference can be larger than the coherence time of the photons, resulting in $T_{\mathrm{d}} \gg T_{\mathrm{c}} \gg T_{\mathrm{p}}$. On the other hand, when the timing resolution of the photon detectors is much shorter than the photon coherence time $\left(T_{\mathrm{c}} \gg T_{\mathrm{d}}\right)$, a high visibility HOM interference can be obtained even if asynchronous photon sources are used. The essential point is that 


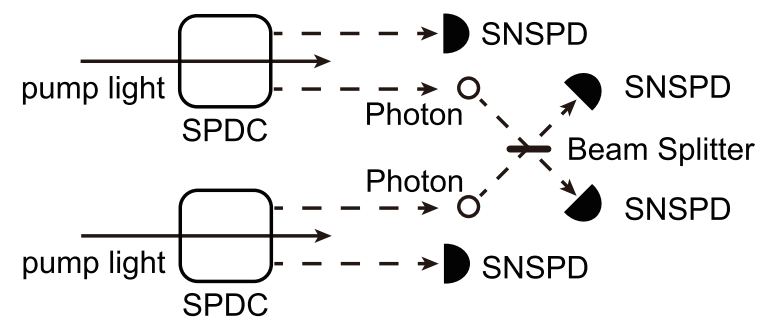

Fig. 2 Schematic of the HOM interference by the timing measurement.

the high timing resolution coincidence detection postselects the events where two photons arrived at the beam splitter within their coherence times. If we use this high precision timing measurement for the synchronous pump light case, we can enhance the visibility by post selection too. SNSPD is the best candidate to implement such a high precision timing measurement. The first experimental demonstration of HOM interference with this timing measurement has been reported in Ref. [31] with the visibility of 0.77 in the case of photon pair source with CW pump laser. Recently, the experimental improvement realizes higher visibility of 0.93 in Ref. [32], [33], which is competitive with the pulsed pump SPDC experiments. The detailed metrology for the analysis of the time resolved coincidence are also shown in Ref. [32]. The visibility will be further improved with lower timing jitter SNSPD and lower pump power. Such a high performance will open up asynchronous photonic quantum information processing that enables synchronization-free long distance quantum communication and quantum computation.

\section{Telecom Quantum Network with Natural Matter Quantum Systems}

The high performance of SNSPD in the range of telecom wavelength is preferable also for a long distance mattermatter entanglement creation through an optical fiber [34]. The creation of the entanglement between distant matters is realized by the quantum teleportation as shown in Fig. 3 a. Individually prepared each atom is first entangled with a photon via matter-photon interaction, for example Raman scattering. The photons from two separated matter systems travel to the middle of those and are performed the Bell measurement, which realizes the quantum teleportation of the photonic states to the matter system used as a storage of the quantum state. The photon loss in the optical fiber reduces the success probability of the Bell measurement, but the notice of the success of the Bell measurement tells us the creation of the entanglement between two distant matter systems. Such a heralded entanglement between distant quantum storages plays an essential role for quantum communication protocols, for example, device independent QKD and quantum repeaters.

The matter quantum storages have been actively studied in the last two decades, but most of the candidates such as $\mathrm{NV}$ center in diamond, $\mathrm{Rb}$ and $\mathrm{Cs}$ atomic ensembles and

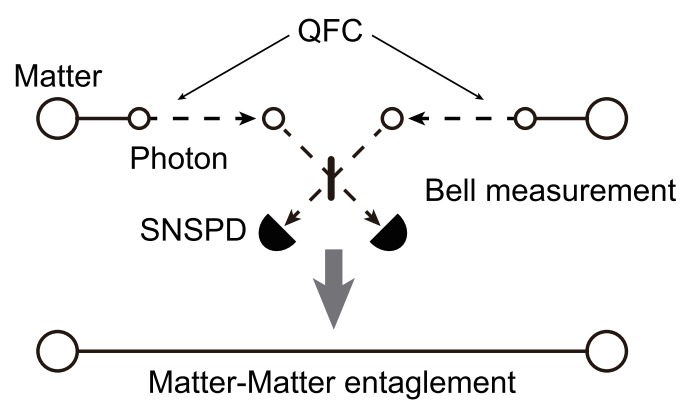

b

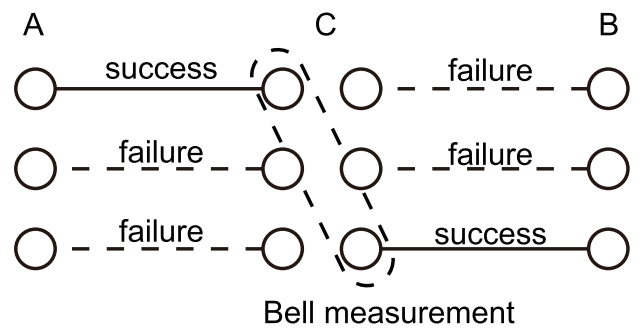

Fig. 3 Schematics of a matter-matter entanglement creation via quantum teleportation and $\mathbf{b}$ quantum repeater protocol

trapped ions have demonstrated the entanglement with photons ranging from $600 \mathrm{~nm}$ to $900 \mathrm{~nm}$, which are far shorter wavelengths than telecom range [35]-[38]. The transmittance of the optical fiber with the length of $l \mathrm{~km}$, which is represented by $T=10^{-\alpha l / 10}$, is significantly low at this wavelength range ( $\alpha=3[\mathrm{~dB} / \mathrm{km}])$. This is not compatible to the telecom range having $\alpha=0.2[\mathrm{~dB} / \mathrm{km}]$, especially for a long distance optical fiber. Recently, to fill this wavelength gap, quantum frequency conversion (QFC) [39] changing the color of photons while preserving the state have been actively studied, too [40]-[43]. Here I focus on QFC realized by the second order nonlinear optical phenomena known as difference frequency generation (DFG).

In the DFG process, the incoming photon having the frequency $\omega_{\mathrm{i}}$ is converted to $\omega_{\mathrm{o}}$ with the strong pump light having $\omega_{\mathrm{p}}$ satisfying $\omega_{\mathrm{i}}=\omega_{\mathrm{o}}+\omega_{\mathrm{p}}$. The waveguide periodically poled lithium niobate (PPLN) has been used for this frequency conversion, and the conversion efficiency inside the crystal up to $70 \%$ with several hundreds $\mathrm{mW}$ pump power has been observed [41]. The average photon numbers in the frequency modes $\omega_{\mathrm{i}}$ and $\omega_{\mathrm{o}}$ are less than unity in the case of QFC. Therefore, scattered photons coming from Raman or parametric interaction with strong pump light cause the background photons in the output frequency mode $\omega_{0}$. In typical experiments, the pump light is a continuous wave having a narrow line width to ensure the coherence time of the process, resulting in the continuous background photons. On the other hand, the input and output photon mode is temporally well-defined. Therefore, the photon detection with a high timing resolution realized by SNSPD is suitable to estimate the intrinsic performance of QFC. In the first experimental demonstration of PPLN based QFC to telecom range [41], due to the low timing resolution and the 
high dark count rate of single photon detection with APD in the telecom range, the reported fidelity was 0.75 . However, the high timing resolution and the low dark count rate of SNSPD [44] reduce the contribution of the background photons and clearly reveal the intrinsic performance of QFC with the fidelity of 0.93 [43].

Applications of the PPLN based QFC and SNSPDs to matter quantum systems have been actively studied. In the case of the cold $\mathrm{Rb}$ atomic ensemble, the preservation of the nonclassical statistics of the emitted photons at $780 \mathrm{~nm}$ during the conversion process to the telecom range has been demonstrated [45], [46]. Furthermore, the atomtelecom photon entanglement has been shown in Ref. [47]. In the case of $\mathrm{Ca}^{+}$ion trap, the single photon having the resonant wavelength of $866 \mathrm{~nm}$ has been converted to a telecom range, and then it has been distributed through $10-\mathrm{km}$ optical fiber [48]. The entanglement preservation has been also shown in Ref. [49]. For Nitrogen-Vacancy (NV) centers in diamond, the possibility of the conversion from its resonant wavelength of $632 \mathrm{~nm}$ to a telecom range has been shown experimentally, as reported recently in Refs. [50] and [51]. All the experiments utilized SNSPDs to see the intrinsic performances of the systems.

\section{Telecom Quantum Network with Artificial Quan- tum Systems}

Not only the matter quantum systems based on naturally existed species in the previous section, but also artificial quantum systems have been studied actively. One of such systems accessible to telecom wavelength photons is a micro/nano-mechanical systems [52]-[54]. Due to the small effective mass of the nano-mechanical oscillator, the thermal noise can be eliminated at the cryogenic temperature. By the interaction between the mechanical oscillator and the light via the radiation pressure, nano-mechanical system is entangled with the photons, similarly to the atom-photon quantum systems in the previous section. Many mechanical oscillators have been studied so far, but the highest mechanical frequency is $\sim \mathrm{GHz}$. Although this frequency range, which is far lower than optical frequency, implies the cooling of the system to $\sim 10 \mathrm{mK}$ to beat the thermal noise, it is attractive that the artificial nano-mechanical system can be directly entangled with the telecom photons. In such experiments [52], [53], the high performance SNSPDs have played an important role to reveal the intrinsic properties of the composite quantum systems consisting of nano-mechanical and photonic systems

\section{Quantum Repeaters}

Future quantum internet [34], [55] aiming at a quantum communication between any two users on the earth will provide a secure communication that is robust against the eavesdropping by a high performance computing including a quantum computer. It prerequisites a quantum repeater [56], [57] that significantly suppresses the ineffi- ciency of the photon transmission through the optical fiber. The photon loss in the optical fiber is a major obstacle for a long distance quantum communication because it has an exponential increase function for the length of the optical fiber. Even in the case of the photon in telecom range, the transmittance for $100 \mathrm{~km}$ fiber becomes around $1 / 100$. In the case of the quantum repeater protocol, the inefficiency can be solved by a parallelism and a collective quantum operations and measurements as shown in Fig. 3 b. Let us suppose that there are multiple transmission line between Alice and Bob, and each transmission line is divided into a certain number of entanglement distributions between the nearest neighbor nodes. At the nodes, the Bell measurement is performed for teleporting the state to the next node. Here we represent the efficiency of the Bell state distribution as $\eta$. The average number of repetition to obtain one Bell state between the nearest neighbor nodes is $1 / \eta$. If only one transmission line is used, the total average number of repetition for Alice and Bob is $(1 / \eta)^{N}$, where $N$ is the number of the division. On the other hand, if the multiple transmission lines are simultaneously used, and the Bell measurement is performed between successfully distributed Bell states, the total average number of Bell states for Alice and Bob is proportional to $1 / \eta$ in an sufficient number of $N$.

In order to implement the Bell measurement on the successful events, most of the protocols assume quantum storages, which reduce the effective number of necessary line $N$, for the preservation of the Bell state until the Bell state distribution succeeds in another side. The matter-photon entanglement described in the previous sections provides the way to share Bell state between quantum storages as shown in Fig. 3 a. The Bell measurement on the protonic qubit notices the success of the sharing the Bell state between the nearest neighbor nodes. High performance SNSPDs are expected to contribute to realize the quantum repeater based on the matter-photon entangled systems. Another way to implement the Bell measurement on the successful events is more straight forward. In the nodes, a special photonic graph state is prepared and measured with the distributed state in multiple transmission line [58], [59]. Due to the specific property of the graph state, the measurement performs Bell measurement only on the survived qubits. In this case, the performance of the photon detector is crucial because the inefficiency of the detector contributes to reduce the effective distribution efficiency $\eta$. Although a necessary detection efficiency to beat against the direct transmission depends on the depth of the error correcting codes, without any codes, the necessary inefficiency will be the order of 0.01 [59]. Therefore, the integrated photonic technologies with SNSPD is attractive to achieve such a high efficiency even in the case of multiplexed photonic circuits.

\section{Conclutions}

This review summarizes elemental photonic QIP tasks with SNSPDs and its application to hybrid quantum systems including diverse matters and photons, which have been ac- 
tively studied recently. In those experiments, the high performance of SNSPDs was indispensable for clearly revealing the quantum properties. I believe the developments of SNSPSs and TESs will also play an important role in future quantum internet that consists of a large scale photonic QIP and matter quantum systems.

\section{Acknowledgments}

This work was supported by CREST, JST JPMJCR1671 and JPMJCR18H1; MEXT/JSPS KAKENHI Grant Number 18H04291, 16H02214, 15KK0164.

\section{References}

[1] R.H. Hadfield and G. Johansson, "Superconducting Devices in Quantum Optics," Springer International Publishing, 2016.

[2] S. Miki, T. Yamashita, Z. Wang, and H. Terai, "A 64-pixel NbTiN superconducting nanowire single-photon detector array for spatially resolved photon detection," Optics Express, vol.22, no.7, pp.78117820, 2014.

[3] C.H. Bennett and G. Brassard, "Quantum cryptography: Public key distribution and coin tossing," Proceedings of IEEE International Conference on Computers, Systems and Signal Processing, pp.175$179,1984$.

[4] A.K. Ekert, "Quantum cryptography based on Bell's theorem," Phys. Rev. Lett., vol.67, no.6, pp.661-663, 1991.

[5] H.-L. Yin, et al., "Measurement-Device-Independent Quantum Key Distribution Over a 404 km Optical Fiber," Phys. Rev. Lett., vol.117, no.19, 190501, 2016.

[6] A. Boaron, et al., "Secure quantum key distribution over $421 \mathrm{~km}$ of optical fiber," arXiv:1807.03222

[7] C.H. Bennett, G. Brassard, C. Crépeau, R. Jozsa, A. Peres, and W.K. Wootters, "Teleporting an unknown quantum state via dual classical and Einstein-Podolsky-Rosen channels," Phys. Rev. Lett., vol.70, no.13, pp.1895-1898, 1993.

[8] D. Bouwmeester, J.-W. Pan, K. Mattle, M. Eibl, H. Weinfurter, and A. Zeilinger, "Experimental quantum teleportation," Nature, vol.390, pp.575-579, 1997.

[9] P.G. Kwiat, K. Mattle, H. Weinfurter, A. Zeilinger, A.V. Sergienko, and Y. Shih, "New High-Intensity Source of Polarization-Entangled Photon Pairs," Phys. Rev. Lett., vol.75, no.24, pp.4337-4341, 1995.

[10] C.K. Hong, Z.Y. Ou, and L. Mandel, "Measurement of subpicosecond time intervals between two photons by interference," Phys. Rev. Lett., vol.59, no.18, pp.2044-2046, 1987.

[11] E. Knill, R. Laflamme, and G.J. Milburn, "A scheme for efficient quantum computation with linear optics," Nature, vol.409, no.6816, pp.46-52, 2001.

[12] M. Koashi, T. Yamamoto, and N. Imoto, "Probabilistic manipulation of entangled photons," Phys. Rev. A, vol.63, no.3, 030301(R), 2001.

[13] T. Yamamoto, M. Koashi, and N. Imoto, "Concentration and purification scheme for two partially entangled photon pairs," Phys. Rev. A, vol.64, no.1, 012304, 2001.

[14] T.B. Pittman, B.C. Jacobs, and J.D. Franson, "Probabilistic quantum logic operations using polarizing beam splitters," Phys. Rev. A, vol.64, no.6, 062311, 2001.

[15] D.E. Browne and T. Rudolph, "Resource-Efficient Linear Optical Quantum Computation," Phys. Rev. Lett., vol.95, no.1, 010501, 2005.

[16] J.-W. Pan, Z.-B. Chen, C.-Y. Lu, H. Weinfurter, A. Zeilinger, and M. Żukowski, "Multiphoton entanglement and interferometry," Rev. Mod. Phys., vol.84, no.2, pp.777-838, 2012.

[17] P. Kok, W.J. Munro, K. Nemoto, T.C. Ralph, J.P. Dowling, and G.J. Milburn, "Linear optical quantum computing with photonic qubits," Rev. Mod. Phys., vol.79, no.1, pp.135-174, 2007.
[18] R. Raussendorf and H.J. Briegel, "A One-Way Quantum Computer," Phys. Rev. Lett., vol.86, no.22, pp.5188-5191, 2001.

[19] R. Raussendorf, D.E. Browne, and H.J. Briegel, "Measurement based Quantum Computation on Cluster States," Phys. Rev. A, vol.68, no.2, 022312, 2003.

[20] A. Politi, M.J. Cryan, J.G. Rarity, S. Yu, and J.L. O'Brien, "Silica-on-silicon waveguide quantum circuits," Science, vol.320, pp.646-649, 2008.

[21] J.W. Silverstone, D. Bonneau, K. Ohira, N. Suzuki, H. Yoshida, N. Iizuka, M. Ezaki, C.M. Natarajan, M.G. Tanner, R.H. Hadfield, V. Zwiller, G.D. Marshall, J.G. Rarity, J.L. O’Brien, and M.G. Thompson, "On-chip quantum interference between silicon photonpair sources," Nat. Photon., vol.8, no.2, pp.104-108, 2013.

[22] B.J. Metcalf, J.B. Spring, P.C. Humphreys, N. Thomas-Peter, M. Barbieri, W.S. Kolthammer, X.-M. Jin, N.K. Langford, D. Kundys, J.C. Gates, B.J. Smith, P.G.R. Smith, and I.A. Walmsley, "Quantum teleportation on a photonic chip," Nat. Photon., vol.8, no.10, pp.770-774, 2014.

[23] J. Carolan, C. Harrold, C. Sparrow, E. Martin-Lopez, N.J. Russell, J.W. Silverstone, P.J. Shadbolt, N. Matsuda, M. Oguma, M. Itoh, G.D. Marshall, M.G. Thompson, J.C.F. Matthews, T. Hashimoto, J.L. O'Brien, and A. Laing, "Universal linear optics," Science, vol.349, no.6249, pp.711-716, 2015.

[24] C. Xiong, X. Zhang, Z. Liu, M.J. Collins, A. Mahendra, L.G. Helt, M.J. Steel, D..-Y. Choi, C.J. Chae, P.H.W. Leong, and B.J. Eggleton, "Active temporal multiplexing of indistinguishable heralded single photons," Nat. Commun., vol.7, 10853, 2016.

[25] T. Kobayashi, R. Ikuta, S. Yasui, S. Miki, T. Yamashita, H. Terai, T. Yamamoto, M. Koashi, and N. Imoto, "Frequency-domain Hong-Ou-Mandel interference," Nat. Photon., vol.10, pp.441-444, 2016.

[26] M. Kues, C. Reimer, P. Roztocki, L.R. Cortés, S. Sciara, B. Wetzel, Y. Zhang, A. Cino, S.T. Chu, B.E. Little, D.J. Moss, L. Caspani, J. Azaña, and R. Morandotti, "On-chip generation of high-dimensional entangled quantum states and their coherent control," Nature, vol.546, pp.622-626, 2017.

[27] J.-W. Pan, D. Bouwmeester, M. Daniell, H. Weinfurter, and A. Zeilinger, "Experimental test of quantum nonlocality in three-photon Greenberger-Horne-Zeilinger entanglement," Nature, vol.403, pp.515-519, 2000.

[28] X.-L. Wang, et al., "Experimental Ten-Photon Entanglement," Phys. Rev. Lett., vol.117, no.21, 210502, 2016.

[29] I. Schwartz, D. Cogan, E.R. Schmidgall, Y. Don, L. Gantz, O. Kenneth, N.H. Lindner, and D. Gershoni, "Deterministic generation of a cluster state of entangled photons," Science, vol.354, no.6311, pp.434-437, 2016.

[30] T. Rudolph, "Why I am optimistic about the silicon-photonic route to quantum computing," APL Photonics, vol.2, no.3, 030901, 2017.

[31] M. Halder, A. Beveratos, N. Gisin, V. Scarani, C. Simon, and H. Zbinden, "Entangling independent photons by time measurement," Nat. Phys., vol.3, pp.692-695, 2007.

[32] Y. Tsujimoto, Y. Sugiura, M. Tanaka, R. Ikuta, S. Miki, T. Yamashita, H. Terai, M. Fujiwara, T. Yamamoto, M. Koashi, M. Sasaki, and N. Imoto, "High visibility Hong-Ou-Mandel interference via a time-resolved coincidence measurement," Optics Express, vol.25, no.11, pp.12069-12080, 2017.

[33] Y. Tsujimoto, M. Tanaka, N. Iwasaki, R. Ikuta, S. Miki, T. Yamashita, H. Terai, T. Yamamoto, M. Koashi, and N. Imoto, "Highfidelity entanglement swapping and generation of three-qubit GHZ state using asynchronous telecom photon pair sources," Scientific reports, vol.8, 1446, 2018.

[34] H.J. Kimble, "The quantum internet," Nature, vol.453, pp.10231030, 2008.

[35] D. Hucul, I.V. Inlek, G. Vittorini, C. Crocker, S. Debnath, S.M. Clark, and C. Monroe, "Modular entanglement of atomic qubits using photons and phonons," Nat. Phys., vol.11 pp.37-42, 2015.

[36] S. Ritter, C. Nölleke, C. Hahn, A. Reiserer, A. Neuzner, M. Uphoff, 
M. Mücke, E. Figueroa, J. Bochmann, and G. Rempe, "An elementary quantum network of single atoms in optical cavities," Nature, vol.484, pp.195-200, 2012.

[37] J. Hofmann, M. Krug, N. Ortegel, L. Gerard, M. Weber, W. Rosenfeld, and H. Weinfurter, "Heralded Entanglement Between Widely Separated Atoms," Science, vol.337, no.6090, pp.72-75, 2012.

[38] B. Hensen, H. Bernien, A.E. Dréau, A. Reiserer, N. Kalb, M.S. Blok, J. Ruitenberg, R.F.L. Vermeulen, R.N. Schouten, C. Abellán, W. Amaya, V. Pruneri, M.W. Mitchell, M. Markham, D.J. Twitchen, D. Elkouss, S. Wehner, T.H. Taminiau, and R. Hanson, "Loophole-free Bell inequality violation using electron spins separated by 1.3 kilometres," Nature, vol.526, pp.682-686, 2015.

[39] P. Kumar, "Quantum frequency conversion," Optics Letters, vol.15, no.24, pp.1476-1478, 1990.

[40] S. Tanzilli, W. Tittel, M. Halder, O. Alibart, P. Baldi, N. Gisin, and H. Zbinden, "A photonic quantum information interface," Nature, vol.437, pp.116-120, 2005.

[41] R. Ikuta, Y. Kusaka, T. Kitano, H. Kato, T. Yamamoto, M. Koashi, and N. Imoto, "Wide-band quantum interface for visible-to-telecommunication wavelength conversion," Nat. Commu., vol.2, 1544, 2011.

[42] K. De Greve, L. Yu, P.L. McMahon, J.S. Pelc, C.M. Natarajan, N.Y. Kim, E. Abe, S. Maier, C. Schneider, M. Kamp, S. Höfling, R.H. Hadfield, A. Forchel, M.M. Fejer, and Y. Yamamoto, "Quantum-dot spin-photon entanglement via frequency downconversion to telecom wavelength," Nature, vol.491, pp.421-425, 2012.

[43] R. Ikuta, H. Kato, Y. Kusaka, S. Miki, T. Yamashita, H. Terai, M. Fujiwara, T. Yamamoto, M. Koashi, M. Sasaki, Z. Wang, and N. Imoto, "High-fidelity conversion of photonic quantum information to telecommunication wavelength with superconducting single-photon detectors," Phys. Rev. A, vol.87, no.1, 010301(R), 2013.

[44] S. Miki, T. Yamashita, H. Terai, and Z. Wang, "High performance fiber-coupled NbTiN superconducting nanowire single photon detectors with Gifford-McMahon cryocooler," Optics Express, vol.21, no.8, pp.10208-10214, 2013.

[45] R. Ikuta, et al., "Heralded single excitation of atomic ensemble via solid-state-based telecom photon detection," Optica, vol.3, pp.12791284, 2016.

[46] P. Farrera, N. Maring, B. Albrecht, G. Heinze, and H. de Riedmatten, "Nonclassical correlations between a C-band telecom photon and a stored spin-wave," Optica, vol.3, no.9, pp.1019-1024, 2016.

[47] R. Ikuta, T. Kobayashi, T. Kawakami, S. Miki, M. Yabuno, T. Yamashita, H. Terai, M. Koashi, T. Mukai, T. Yamamoto, and N. Imoto, "Polarization insensitive frequency conversion for an atomphoton entanglement distribution via a telecom network," Nat. Commun., vol.9, 1997, 2018.

[48] T. Walker, K. Miyanishi, R. Ikuta, H. Takahashi, S.V. Kashanian, Y. Tsujimoto, K. Hayasaka, T. Yamamoto, N. Imoto, and M. Keller, "Long-distance single photon transmission from a trapped ion via quantum frequency conversion," Phys. Rev. Lett., vol.120, no.20, 203601, 2018.

[49] M. Bock, P. Eich, S. Kucera, M. Kreis, A. Lenhard, C. Becher, and J. Eschner, "High-fdelity entanglement between a trapped ion and a telecom photon via quantum frequency conversion," Nat. Commun., vol.9, 1998, 2018.

[50] R. Ikuta, et al., "Frequency downconversion of $637 \mathrm{~nm}$ light to the telecommunication band for non-classical light emitted from NV centers in diamond," Optics Express, vol.22, pp.11205-11214, 2014.

[51] A. Dréau, A. Tcheborateva, A.E. Mahdaoui, C. Bonato, and R. Hanson, "Quantum frequency conversion to telecom of single photons from a nitrogen-vacancy center in diamond," Phys. Rev. Applied, vol.9, 064031, 2018.

[52] S. Hong, R. Riedinger, I. Marinković, A. Wallucks, S.G. Hofer, R.A. Norte, M. Aspelmeyer, and S. Gröblacher, "Hanbury Brown and Twiss interferometry of single phonons from an optomechanical resonator," Science, vol.358, no.6360, pp.203-206, 2017.

[53] R. Riedinger, A. Wallucks, I. Marinković, C. Löschnauer, M. Aspelmeyer, S. Hong, and S. Gröblacher, "Remote quantum entanglement between two micromechnical oscillators," Nature, vol.556, pp.473-477, 2018.

[54] M. Asano, Y. Takeuchi, W. Chen, Ş.K. Özdemir, R. Ikuta, N. Imoto, L. Yang, and T. Yamamoto, "Observation of optomechanical coupling in a microbottle resonator," Laser \& Photonics Reviews, vol.10, no.4, pp.603-611, 2016.

[55] S. Wehner, D. Elkouss, and R. Hanson, "Quantum internet: A vision for the road ahead," Science, vol.362, no.6412, eaam9288, 2018.

[56] H.-J. Briegel, W. Dür, J.I. Cirac, and P. Zoller, "Quantum repeaters: The role of imperfect local operations in quantum communication," Phys. Rev. Lett., vol.81, pp.5932-5935, 1998.

[57] L.-M. Duan, M.D. Lukin, J.I. Cirac, and P. Zoller, "Long-distance quantum communication with atomic ensembles and linear optics," Nature, vol.414, pp.413-418, 2001.

[58] K. Azuma, K. Tamaki, and H.-K. Lo, "All-photonic quantum repeaters," Nat. Commun., vol.6, 6787, 2015.

[59] Y. Hasegawa, R. Ikuta, N. Matsuda, K. Tamaki, H-K. Lo, T. Yamamoto, K. Azuma, and N. Imoto, "Experimental timereversed adaptive Bell measurement towards all-photonic quantum repeaters," Nat. Commun., vol.10, 378, 2019.

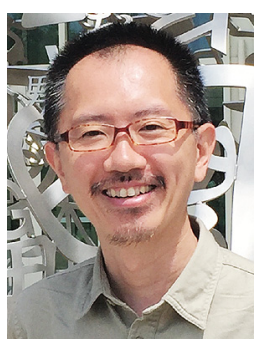

Takashi Yamamoto is a professor at Osaka University, Japan, in the Department of Materials Engineering Science, Graduate school of Engineering Science. He also worked as an associate professor and an assistant professor at Osaka University. He received his M.Sc. degree in physics from Kumamoto University in 1999 and Ph.D. degree from SOKENDAI in 2003. His research interests include fundamental quantum physics, photonics quantum computation and communication. 